\title{
DGNSS Cooperative Positioning in Mobile Smart Devices: a Proof of Concept
}

\author{
Alex Minetto, Member, IEEE, Maria Chiara Bello, and Fabio Dovis, Member, IEEE,
}

\begin{abstract}
In recent years positioning and navigation capabilities in mobile devices have become essential to the evergrowing number of position-related smart applications. Global Navigation Satellite System (GNSS) constitutes the main provider for geo-localization. Therefore consumer-grade, embedded GNSS receivers have become ubiquitous in mobile smart devices. Among these, smartphones play a dominant role in enabling such modern services based on the position information. However, GNSS positioning shows several weaknesses in urban contexts where mobile smart devices are massively diffused. Indeed, the limited sky visibility and multipath scattering induced by buildings severely threaten the quality of the final solution. Two main ingredients can enable novel collaborative strategies capable to increase the robustness of GNSS navigation: The availability of raw GNSS measurements which have been recently disclosed in ultra-lowcost smartphone chipsets and the ubiquitous connectivity provided by modern low-latency, network infrastructures allowing for the near-real-time exchange of data. This work presents the architecture of a Proof Of Concept designed to demonstrate the feasibility of a GNSS-only Cooperative Positioning among networked smartphones equipped with GNSS receivers. The test campaign presented in this work assessed the feasibility of the approach over 4G/LTE network connectivity and an average accuracy improvement over the $40 \%$ w.r.t. to the GNSS standalone solution.
\end{abstract}

Index Terms-Collaborative systems, cooperative positioning, measurements, mobile agents, multi-agent systems, state estimation, pose estimation

\section{INTRODUCTION}

$\mathbf{U}$ P to 10 billion electronic devices with localization capabilities will populate the world by 2025 to satisfy the booming request for Location Based Services (LBS) [1]. While professional Global Navigation Satellite Systems (GNSS) receivers will keep dominating the market of highly precise, accurate, and reliable positioning and navigation, mass-market products and services will demand smart solutions to obtain the best performance through low-cost consumer electronics. A considerable number of LBS such as vehicles pooling, sharing, and remote control of Unmanned Aerial Vehicles (UAV)s are based primarily on the positioning solution provided by mobile mass-market devices (i.e. low-cost and ultra-low-cost embedded GNSS receivers) [2]-[4].

In the last decades, a remarkable research effort has been broadly spent in improving navigation and positioning performance by mostly addressing sensor fusion to GNSS [5],

Manuscript received $[\mathrm{XXX}]$; revised $[\mathrm{XXX}]$; accepted $[\mathrm{XXX}]$. Date of publication $[\mathrm{XXX}]$; date of current version $[\mathrm{XXX}]$. This work was supported by the European Space Agency (ESA) under ESA contract number 4000126230/18/NL/CRS. The authors are with the Department of Electronics and Telecommunications (DET), Politecnico di Torino, Turin, Italy, e-mail: name.surname@polito.it (Corresponding authors: Alex Minetto, Fabio Dovis).
[6]. Advanced integration schemes foreseeing tight and ultratight fusion of GNSS/Inertial Navigation Sytem (INS) measurements offer promising performance in harsh environment where standalone GNSS is typically weak [7]-[10], but they require reliable sensors and their implementation can be infeasible, challenging or not suitable for consumer mobile devices [11]-[14]. As a complementary solution, the sensing of the surrounding environment has become a relevant aid to the navigation and it can be accomplished through vision systems (i.e. cameras [3], [15]) or through several exteroceptive sensors such as Ultra-Wide Band (UWB) units, Light Detection and Ranging (LiDAR) and Ultrasonic Ranging System (URS). Indeed, when inertial sensing is not available or sufficiently reliable, alternative measurements can be used to retrieve information about the surrounding environment such as the distances from anchor points whose position is known [16][18] or by considering other devices as anchors of opportunity, thus relying on their position estimates [19]. Therefore, the contribution of exteroceptive sensors to improve absolute and relative localization has been extensively explored in literature [20]. However, many of the aforementioned high-accuracy technologies are still impracticable for small-sized, low-cost consumer devices and the unavailability of possible anchors in Line of Sight (LOS) or the limited coverage of the sensors can limit the effectiveness of such approaches.

The GNSS can be used to determine relative distances through the exchange of position-related measurements. Since the rise of professional applications, hi-end GNSS receivers have provided such data to mostly support precise positioning (e.g., Real Time Kinematic (RTK)) through the implementation of external corrections. However, in order to perform Cooperative Positioning (CP) among low-cost receivers, lowlevel GNSS data, a.k.a. GNSS raw measurements, have to be accessible in such a class of devices. By coupling the disclosure of raw GNSS data in smartphones and tablets and the recent advances in communication networks (e.g. UltraReliable and Low-Latency Communications (URLLC) in $5 \mathrm{G}$ $\mathrm{NR}$ ), the concept of networked GNSS receiver is expected to rise to mass-market and IoT applications [21]. This trend enables the exchange and integration of satellite-based relative measurements among connected devices, thus the implementation of $\mathrm{CP}$ solutions.

The implementation proposed in this paper foresees the mutual exchange of GNSS raw measurements obtained from integrated ultra-low-cost receivers for a near-real-time computation of the inter-agent distances. The cooperation improves the quality of their positioning through a hybrid navigation scheme proposed in [22], that integrates such auxiliary interagent distances in a peer-to-peer fashion. A demonstrator was 
developed in the context of the European Space Agency (ESA) HANSEL project to assess the feasibility and the effectiveness of GNSS-based CP using consumer-grade smartphones [23], by relying on their access to raw GNSS measurements. This study investigates the feasibility of the proposed paradigm considering a pairwise communication between two devices. Therefore, the analysis of the scalability of the proposed solution is meant out of the scope of this article and it will be addressed in future works.

The outline of the paper is as follows. A set of relevant pioneering works and previous contributions on the topic are discussed in Section II. Theoretical fundamentals on standalone GNSS and CP estimation are recalled in Section III. An overall description of the $\mathrm{CP}$ framework and its implementation is then provided in Section IV. The experimental setup exploited for the preliminary test campaign and the key performance metrics for the evaluation of the positioning performance are described in Section V. The most relevant results in terms of feasibility are discussed in Section VI and eventually, conclusions are drawn along with future investigations in Section VII.

\section{Previous CONTRIBUtions}

The design and development of the proposed Proof Of Concept (PoC) were inspired by a set of relevant research works concerning the use of differential GNSS measurements in cooperative contexts and the use of GNSS in mobile smart devices.

\section{A. Estimation and integration of GNSS Differential range measurements}

In the framework of Differential GNSS (DGNSS) and RTK, previous works investigated the computation of the distance among GNSS receivers by exchanging GNSS measurements only. For example in [24], the authors presented a technique to compute inter-vehicular range measurements through weighted GNSS Double Differences (WDD). Other DGNSSbased techniques are comparatively investigated in [25]. A further method was then proposed by relying on the exchange of both pseudorange measurements and the terms of the Direction Cosine Matrix (DCM) computed within the Position Time Velocity (PVT), in [26].In previous contributions, researchers observed remarkable improvement in $\mathrm{CP}$ by tightly integrating Double Differences (DD), and the quantity of information carried by such differential measurements was demonstrated in [22], [27]-[29].

Pioneering works on the tight integration of Differential GPS (DGPS) and INS in an Hybrid Extended Kalman Filter (EKF)-based positioning algorithm were proposed to improve accuracy and availability of Global Positioning System (GPS) positioning [20], [30], [31]. A two-steps positioning algorithm has been designed to refine the GNSS-only position estimates through a Maximum Likelihood (ML) approach by constraining the positioning solution through inter-vehicle ranges obtained by weighted double differentiation of shareable pseudorange measurements [32]. The approach foresees a distributed computation of the locations of all the agents by each agent. A refined solution was then performed as a weighted mean of the different estimates provided by the collaborating agents (i.e. loosely coupling). The scheme proposed in [32] does not exploit the dynamics of the agents, thus being possibly suboptimal in kinematic applications.

\section{B. GNSS in Mobile Smart Devices}

To support and encourage the rapid innovation trends involving LBS indeed, in 2016, GNSS measurements have been officially disclosed for a given set of consumer smartphones and tablets. Raw GNSS measurements became available to the developers directly from the enabled GNSS chipsets embedded in such mobile devices. The measurements are exposed by enabled on-board GNSS chipsets through proper Application Program Interface (API), thus allowing custom implementations of navigation algorithm and so improved positioning and navigation performance [33]. Thanks to this innovation, several recent works successfully investigated GNSS positioning in smartphones. A controlled scenario analysis was performed on a set of different mobile devices in [34], dual-frequency GNSS positioning solutions has been recently investigated in [35] and the migration of DGNSS and RTK positioning has been proposed for smartphones in several contributions [36][40] as well as Precise Point Position (PPP) [41]-[44]. On one hand, such analysis often emphasized technical and technological limits of this market segment such as bad antenna design and poor quality of code pseudorange measurements while on the other hand, they opened a variety of potential solutions for the improvement of positioning and navigation capabilities in consumer electronics.

\section{THEORETICAL BACKGROUND}

Fundamentals on standalone GNSS and CP estimation are briefly recalled hereafter to clarify the theoretical solution used to achieve an effective $\mathrm{CP}$ algorithm.

\section{A. Positioning Problem in GNSS}

Positioning and navigation in GNSS can be treated as a discrete-time estimation process. In fact, by considering a snapshot of a generic kinematic object taken at the time instant $t_{k}$, an ideal single point GNSS-only PVT solution is defined as a $8 \times 1$ column state vector

$$
\boldsymbol{\theta}_{k}=\left[\begin{array}{lll}
\mathbf{x}_{k}^{\top} & \mathbf{v}_{k}^{\top} & \mathbf{b}_{k}^{\top}
\end{array}\right]^{\top}
$$

where the variables

$$
\mathbf{x}_{k}=\left[\begin{array}{c}
x_{k} \\
y_{k} \\
z_{k}
\end{array}\right], \mathbf{v}_{k}=\left[\begin{array}{c}
\dot{x}_{k} \\
\dot{y}_{k} \\
\dot{z}_{k}
\end{array}\right], \mathbf{b}_{k}=\left[\begin{array}{c}
\delta b_{k} \\
\delta \dot{b}_{k}
\end{array}\right]
$$

describe respectively the set of Cartesian location coordinates and the related axial velocities according to a given reference frame (e.g. East-North-Up), as well as the receiver clock bias and its drift w.r.t. a coordinated time scale (i.e. GNSS time).

GNSS receivers provide an estimate of (1) based, in turn, on the estimation of the Time of Flight (ToF) and the Doppler shift (w.r.t. the nominal carrier frequency) of the navigation 


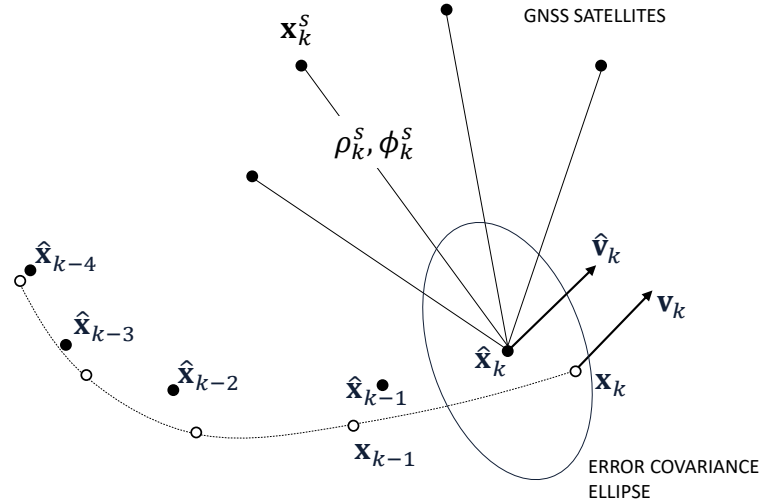

Fig. 1: Trilateration principle applied to GNSS single point positioning estimation. True positions, $\mathbf{x}_{k}$ (white dots) are compared to estimated positions, $\hat{\mathbf{x}}_{k}$ (black dots). The error covariance ellipse is a graphical representation of the statistical uncertainty described by $\mathbf{P}_{k}$.

signals transmitted by GNSS satellites [45]. The first, multiplied by the speed of light, returns a quantity referred as pseudorange measurements, $\rho_{k}^{s}$, which is defined according to

$$
\rho_{k}^{s}=r_{k}^{s}+c \cdot \delta b_{k}+\delta t_{D}
$$

where $\delta b_{k}$ is the clock bias term included in $\mathbf{b}_{k}(1)$ and $\delta t_{D}$ collects any unwanted additional biases due to ionospheric and tropospheric effects acting on the Radio-Frequency (RF) signal, as well as the delays introduced by the receiver hardware and multipath scattering. Prior to the PVT computation, raw pseudoranges and doppler measurements are corrected leading to an estimate of the satellites-to-receiver geometrical distances and the associated changing rates, namely pseudorange and pseudorange rate, respectively. The pseudoranges are corrected by the receiver clock bias computed at $t_{k-1}$, the relativistic correction and the satellite clock error estimated through the ephemeris data

$$
\hat{\boldsymbol{\rho}}_{k}^{N}=\boldsymbol{\rho}_{k}-\delta b_{k-1}+d t_{k-1}^{s} \cdot c
$$
drift

while the pseudoranges rates are corrected by the user clock

$$
\hat{\phi}_{k}=\phi_{k}-\delta \dot{b}_{k-1} .
$$

Such a set of observable measurements is defined as a $2 S \times 1$ column vector

$\mathbf{z}_{k}=\left[\begin{array}{c}\hat{\mathbf{r}}_{k} \\ \hat{\boldsymbol{\phi}}_{k}\end{array}\right]=\left[\begin{array}{llllllll}\hat{r}_{k}^{1} & \hat{r}_{k}^{2} & \ldots & \hat{r}_{k}^{S} & \hat{\phi}_{k}^{1} & \hat{\phi}_{k}^{2} & \ldots & \hat{\phi}_{k}^{S}\end{array}\right]^{\top}$

where the generic $\hat{r}_{k}^{s}$ and $\hat{\phi}_{k}^{s}$ are the range and Doppler estimates associated to the $s$-th satellite and $S$ is the total number of satellite used for the PVT. A $2 S \times 2 S$ measurements covariance matrix, $\mathbf{R}_{k}=\mathrm{E}\left[\left(z_{k}-\mathrm{E}\left(z_{k}\right)\right)\left(z_{k}-\mathrm{E}\left(z_{k}\right)\right)^{\top}\right]$, defines the quality and possible cross-correlations of the observables. The output of the PVT stage typically includes an estimate of (1), and of the associated $N \times N$ covariance matrix $\mathbf{P}_{k}=\mathrm{E}\left[\left(\theta_{k}-\mathrm{E}\left(\theta_{k}\right)\right)\left(\theta_{k}-\mathrm{E}\left(\theta_{k}\right)\right)^{\top}\right]$ which characterizes the statistical uncertainty of the solution. Such an uncertainty is due, in turn, to the residual noise affecting the estimation of satellite-to-receiver range, namely User Equivalent Range Error (UERE). Both the UERE and the geometry of the visible satellites negatively affect the precision of the estimated solution [45].

A linear estimator such as Weighted Least Square (WLS) can be first used to solve for a linearized trilateration problem exploiting four or more satellite-to-receiver measurements [45], as show in Figure 1. A consolidated iterative strategy allow to solve for a differential quantity w.r.t. to a known reference state vector (i.e. $\theta_{k}^{*}=\mathbf{0}_{N \times 1}$ )

$$
\Delta \boldsymbol{\theta}_{k}=\left(\mathbf{H}_{k}^{\top} \mathbf{R}_{k}^{-1} \mathbf{H}_{k}\right)^{-1}-\mathbf{H}_{k} \mathbf{R}_{k}^{-1} \Delta \hat{\mathbf{r}}_{k}
$$

where the modified DCM also referred to observation matrix, $\mathbf{H}_{k}$, is built through the known satellite positions and the linearization point, $\mathbf{x}_{k}^{*}$, according to

$$
\mathbf{H}_{k}=\left[\begin{array}{cccc}
h_{x, k}^{1} & h_{y, k}^{1} & h_{z, k}^{1} & 1 \\
h_{x, k}^{2} & h_{y, k}^{2} & h_{z, k}^{2} & 1 \\
\vdots & \vdots & \vdots & \vdots \\
h_{x, k}^{S} & h_{y, k}^{S} & h_{z, k}^{S} & 1
\end{array}\right]
$$

where the first three elements of the $s$-th row constitute the unitary steering vector, $\mathbf{h}_{k}^{s}=\left[\begin{array}{lll}h_{x, k}^{s} & h_{y, k}^{s} & h_{z, k}^{s}\end{array}\right]$, directed towards the $i$-th satellites

$$
h_{x, k}^{s}=\frac{x_{k}^{s}-x^{*}}{r_{k}^{s}} \quad h_{y, k}^{s}=\frac{y_{k}^{s}-y^{*}}{r_{k}^{s}} \quad h_{z, k}^{s}=\frac{z_{k}^{s}-z^{*}}{r_{k}^{s}}
$$

and $x^{*}, y^{*}, z^{*}$ are the coordinates of the linearization point $\mathbf{x}_{k}^{*}$. Equation (7) can be iteratively refined up to the convergence of the solution. An estimate of the covariance of the solution is hence $\hat{\mathbf{P}}_{k}=\left(\mathbf{H}_{k}^{\top} \mathbf{R}_{k}^{-1} \mathbf{H}_{k}^{\top}\right)^{\top} \mathbf{H}_{k}^{\top} \mathbf{R}_{k}^{-1}$. Once a first state estimate, $\hat{\boldsymbol{\theta}}_{k}$, is achieved, linear or non-linear Bayesian filters are typically implemented to estimate both $\boldsymbol{\theta}_{k}$ and $\mathbf{P}_{k}$ by taking into account some a-priori knowledge about the model of the system dynamics [46]. Limited visibility of the GNSS satellites and high-variance of the UEREs in the pseudorange estimation lead to low-precision state estimation while unpredictable biases are responsible for a degraded accuracy of the solution. Such conditions are often verified in urban environments where a relevant part of consumer, lowcost receivers operate on a daily basis.

\section{B. Cooperative Positioning Integrating Inter-agent Distances}

A valuable strategy to cope for the aforementioned GNSS weaknesses is to integrate auxiliary ranging measurements obtained by further anchor points other than the GNSS satellites, as it was introduced in [47]. A generic terrestrial range measurement between ground receivers $a$ and $b$, is referred hereafter to as inter-agents distance and it can be ideally defined as the Euclidean norm $d_{a b, k} \triangleq\left\|\mathbf{d}_{a b, k}\right\|$ where the inter-agent vector, a.k.a. baseline vector, $\mathbf{d}_{a b, k}$ is defined as

$$
\mathbf{d}_{a b, k}=\left[\begin{array}{c}
x_{a, k}-x_{b, k} \\
y_{a, k}-y_{b, k} \\
z_{a, k}-z_{b, k}
\end{array}\right]=\left[\begin{array}{c}
\Delta x_{a b, k} \\
\Delta y_{a b, k} \\
\Delta z_{a b, k}
\end{array}\right]
$$




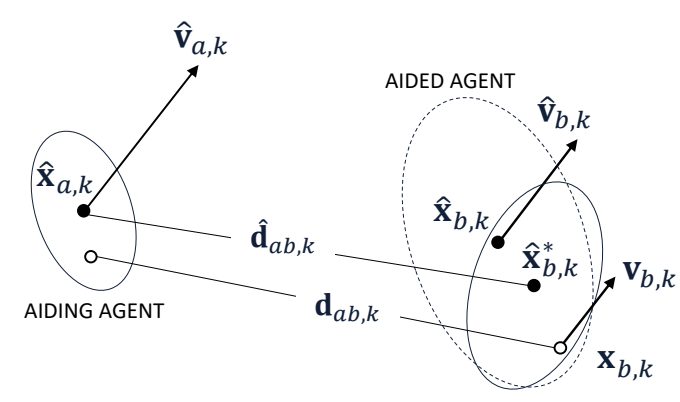

Fig. 2: Graphical representation of pairwise collaborative single point positioning estimation exploiting inter-agent range measurements.

Intuitively, the true baseline length corresponds to the Euclidean distance between the true positions $\mathbf{x}_{a, k}$ and $\mathbf{x}_{b, k}$.

By considering synchronous agents in real conditions, we assume to obtain an estimate $\hat{\mathbf{d}}_{a b, k}$ using estimated input quantities (e.g. position estimates or pseudorange measurements). All the inter-agents measurements broadly employed in different $\mathrm{CP}$ approaches are expected to bring additional information to the positioning problem. Therefore, they cannot be dependent on the positioning estimate itself and for the same reason, the Euclidean distance between the GNSS standalone position estimates is not suitable for providing auxiliary information on the state unknowns in (1), through a GNSS-based approach. Furthermore, the Euclidean norm, a.k.a. Absolute Position Difference (APD) does not foresee common error cancellation on the input measurements [48]. Therefore, this study relies on the implementation of the WDD algorithm mentioned in Section II-A, whose theoretical aspects are recalled in Section III-C. By assuming that DGNSS-based inter-agent distances, $\hat{\mathbf{d}}_{a b, k}$, are independent and uncorrelated w.r.t. the position estimates $\hat{\mathbf{x}}_{a, k}, \hat{\mathbf{x}}_{b, k}$ and, by assuming that $b$ shows better performances in terms of accuracy and precision w.r.t. $a$, the inter-agent distance can be integrated to improve the state estimation of receiver $a$, thus reducing its bias and covariance [49], [50] hence improving both accuracy and precision as depicted in Figure 2.

\section{DGNSS Inter-agent Distance Estimation}

Due to the continuous motion of the satellites and the potential kinematics of the receivers, the estimation of DGNSS inter-agent distances requires an accurate synchronization of the measurements in order to be properly combined.

1) Synchronization of the observable measurements: In general, it can be assumed that two generic receivers, $a$ and $b$, provide the dump of the observable measurements and the related state estimation according to two independent and discrete time axis that will be indicated through the discrete indexes $k$ and $l$, respectively. As shown in Figure 3, these instants can be mapped with a given accuracy to the GNSS time scale according to the estimated clock biases $\delta b_{k}, \delta b_{l}$ obtained at $t_{k}, t_{l}$.

According to the reception time of each satellite, $t_{R X, a}$, a set of observable measurements $\mathbf{z}_{k}$ is dumped by the

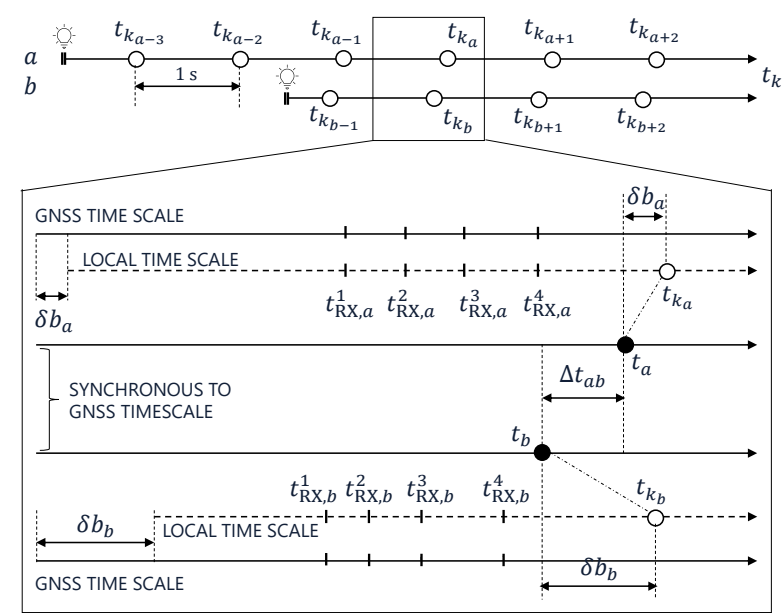

Fig. 3: Asynchronous measurements and PVT epochs in independent GNSS receivers.

receiver $a$ at $t_{k}$, referred to its local time axis. In order to provide a differential estimation of $d_{a b, k}$, raw pseudorange measurements included in $\mathbf{z}_{l}$ provided by receiver $b$ must be consistent, so they have to be dumped at the same time instant, $t_{k}$. This is not granted by standalone GNSS receivers since they are not expected to be synchronous.

Therefore, even if observable measurements and PVT solutions are dumped and time-tagged w.r.t. to the common GNSS time-scale, they refer to different time instants $t_{a}^{(k)}$ and $t_{b}^{(k)}$, with an offset $\Delta t_{a b}^{(k l)}=t_{a}^{(k)}-t_{b}^{(l)}$, as depicted in Figure 3.

It is helpful to remark that the sign of the offset depends on such GNSS timestamps, formally $\Delta t_{a b}^{(k l)}=-\Delta t_{b a}^{(l k)}$.

This offset can be effectively compensated in low-dynamics scenarios (i.e. pedestrian applications) through the Doppler measurements $\hat{\phi}_{k}$ thus aligning in time raw pseudorange measurements shared among collaborating receivers [51], through

$$
\begin{aligned}
\hat{\rho}_{b, k}^{s} & =\hat{\rho}_{b}\left(t_{a}^{(k)}\right)=\hat{\rho}_{b}^{s}\left(t_{b}^{(j)}\right)+\Delta t_{a b}^{(k l)} \\
& =\rho_{b}^{s}\left(t_{b}^{(l)}\right)+\Delta t_{a b}^{(k l)} \cdot \lambda \cdot \phi_{a}^{s}\left(t_{a}^{(k)}\right) .
\end{aligned}
$$

In order to effectively apply (11) dump epochs must be chosen with minimum delay to preserve the validity of the linear model in 11, and the timestamps $t_{a}^{(k)}, t_{b}^{(l)}$ of the measurements must be accurately known w.r.t. the common GNSS reference scale.

2) Double Difference Ranging: Once the time offset is compensated, DGNSS algorithms can be used to determine inter-agent distance.

Assuming the receiver $a$ as the target of this analysis, the time notation can be simplified. Time instants, $t_{a}^{(k)}$, referred to the GNSS time-scale are hereafter referred to a general dump epochs $t_{k}$, and all the observable measurements provided by the receiver $b$ are assumed aligned in time to the measurements dumped by $a$ at $t_{k}$.

Whenever two generic satellites $r$ and $s$ are visible to both the receivers and the two pair of measurements can be shared and synchronized, a DD measurement can be obtained as 
the difference of two Single Differences (SD)s between such pseudorange measurements

$$
D_{a b, k}^{s r}=S_{a b, k}^{s}-S_{a b, k}^{r}=\Delta R_{a b, k}^{s r}+\Sigma_{a b, k}
$$

where $S_{a b, k}^{s}$ is a single difference computed as $S_{a b, k}^{s}=$ $\rho_{a, k}^{s}-\rho_{b, k}^{s}$ while $\Sigma_{a b, k}$ is a random variable collecting residual uncorrelated error contributions such as multipath, secondorder noise components of the receiver front-ends and additional non-modelled noise contributions [45]. Figure 4 shows a pictorial representation of differential ranging between a pair of receivers sharing measurements about 5 visible satellites. The term $\Delta R^{s r}$ can be expressed highlighting the dependency from the baseline vector as

$$
\Delta R_{k}^{s r}=\left[\mathbf{h}_{a, k}^{s}-\mathbf{h}_{b, k}^{r}\right]^{T} \mathbf{d}_{a b, k}
$$

where $\mathbf{h}_{m}^{s}$ is a unitary steering vector defined through the displacement vector between satellite and receiver estimated positions [45]. The computation of DD measurements can be obtained from the linear relationship $\mathbf{D}_{a b, k}=\mathbf{L}_{D D} \mathbf{S}_{a b, k}$, as

$$
\underbrace{\left[\begin{array}{c}
D_{a b, k}^{12} \\
D_{a b, k}^{13} \\
\vdots \\
D_{a b, k}^{1 S}
\end{array}\right]}_{D D}=\underbrace{\left[\begin{array}{ccccc}
-1 & 1 & 0 & \cdots & 0 \\
-1 & 0 & 1 & \ddots & 0 \\
\vdots & \vdots & \ddots & \ddots & \vdots \\
-1 & 0 & \cdots & \cdots & 1
\end{array}\right]}_{\mathbf{L}_{D D}} \underbrace{\left[\begin{array}{c}
S_{a b, k}^{1} \\
S_{a b, k}^{2} \\
S_{a b, k}^{3} \\
\vdots \\
S_{a b, k}^{S}
\end{array}\right]}_{S D} .
$$

Equation (13) can be then expanded, neglecting the noise contribution in (12), in

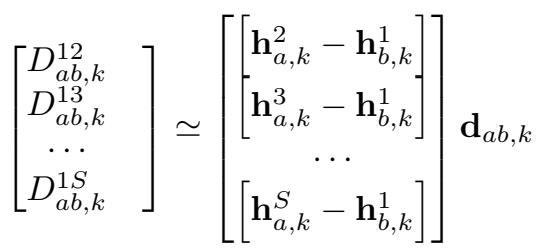

collecting $S-1$ double difference measurements from a set of $S$ satellites simultaneously visible to $a$ and $b$. This set of equations can be exploited to estimate $\mathbf{d}_{a b, k}$ through a WLS algorithm in the form of

$$
\hat{\mathbf{d}}_{a b, k}=\left(\mathbf{H}_{D, k}^{\top} \mathbf{H}_{D, k}\right)^{-1} \mathbf{H}_{D, k}^{\top} \mathbf{D}_{a b, k}
$$

where $\mathbf{D}_{a b, k}$ and $\mathbf{H}_{D, k}$ are respectively the first and the second term in (15). By relying on the estimated covariance matrix of the pseudorange measurements, it is possible to compute the covariance of $\mathbf{D}_{a b, k}$ and implement a weighted baseline estimation as for the WLS shown in (7).

\section{EKF Hybridization for CP tight integration}

In order to integrate the inter-agent distances, (6) can be extended including $\mathbf{d}_{a b, k}$. Collaborative measurements can be indeed included in the initialization stage of both linear estimation (i.e. WLS) and the subsequent Bayesian estimation. PVT algorithm including collaborative range measurements is referred hereafter as Hybrid PVT (H-PVT).

First, a Hybrid Weighted Least Square (H-WLS)-based PVT is performed through a Self-Adaptive Iterative Algorithm (SAIA) proposed in [26] to cope with strong linear

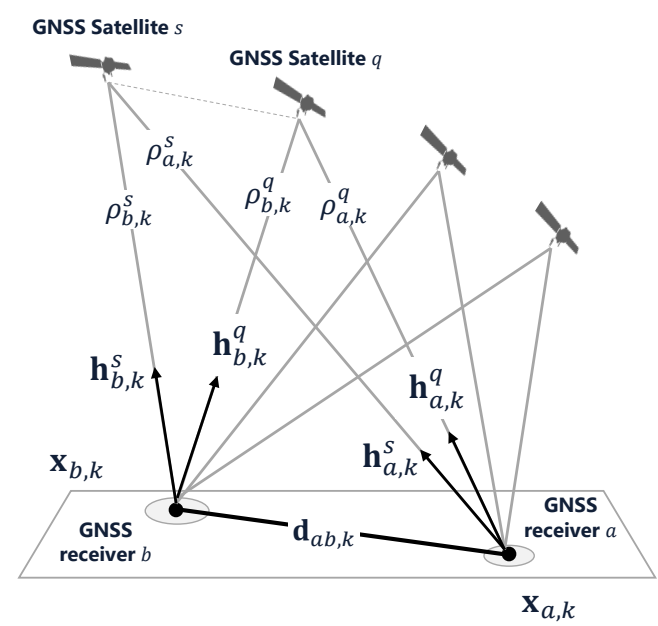

Fig. 4: DGNSS ranging applied between two GNSS receivers sharing measurements of five GNSS satellites. The dashed line represents the combination of single differences obtained w.r.t. generic satellites $s, q$.

dependence of multiple inter-agent distances, thus to solve for ill-conditioned set of linear equations. Once the first fix is obtained, an indirect formulation of the EKF with periodic WLS re-initialization is in charge of a Bayesian positioning estimation, according to the scheme in Figure 5.

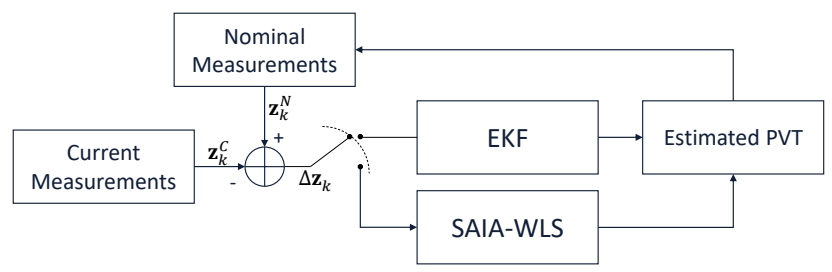

Fig. 5: H-PVT functional block including Indirect EKF scheme fed by SAIA-WLS for reinitialization.

Indirect filtering can be structured as a feed-forward or a feedback EKF [46]. In a feed-forward approach, if the errors injected in the filter are kept small, the linear dynamics model is acceptable. While, if they become larger, the errors estimated by the EKF will drift with time. For this reason in navigation applications that integrate INS, a feedback loop is added to mitigate this drawback [52]. In the implementation of the PoC, inertial sensors are not considered, therefore the error is estimated as the difference between the current measurements exposed by the receiver's post-correlation stage and the nominal ones, which are evaluated by applying the corrections found in the previous step, according to Figure 5. Furthermore, to avoid the error drift, a reset can be periodically performed, by assigning infinite value to the terms of the model noise covariance matrix or equivalently, using a H-WLS PVT evaluation instead of the Bayesian estimation routine.

In the indirect filtering, the prediction step is fed with a zero-state vector, $\boldsymbol{\theta}_{k}=\mathbf{0}_{1 \times N}$. The a priori error covariance matrix $\mathbf{P}_{k}^{-}$and the Kalman gain $\mathbf{K}_{k}$ are computed according 
to the legacy Kalman equations [46]. Differently from the direct formulation, in this case, $\Delta \mathbf{z}_{k}$ is the difference between the current measurements and the nominal measurements

$$
\Delta \hat{\mathbf{z}}_{k}=\left[\begin{array}{lll}
\Delta \boldsymbol{r}_{k} & \Delta \boldsymbol{\phi}_{k} & \Delta \boldsymbol{d}_{k}
\end{array}\right]^{\top}
$$

where

$$
\begin{aligned}
\Delta \boldsymbol{\rho}_{k} & =\Delta \boldsymbol{r}_{k}^{N}-\Delta \boldsymbol{r}_{k}^{C} \\
\Delta \phi_{k} & =\Delta \boldsymbol{\phi}_{k}^{N}-\Delta \boldsymbol{\phi}_{k}^{C} \\
\Delta \boldsymbol{d}_{k} & =\Delta \boldsymbol{d}_{k}^{N}-\Delta \boldsymbol{d}_{k}^{C}
\end{aligned}
$$

Current measurements are the pseudorange measurements vector, $\rho_{k}$, and $\phi_{k}$, the measurement vector including Doppler measurements. Nominal measurements are instead the measured pseudoranges and pseudoranges rates with corrections found at state $\hat{\theta}_{k-1}$.

The approximation state in indirect filtering is the state estimated as

$$
\boldsymbol{\theta}_{k}^{*}=\boldsymbol{\theta}_{k-1}
$$

By adding the estimated value to the previous state as for iterative WLS solutions, the current state $\boldsymbol{\theta}_{k}$ is found.

\section{CPA/CPS WITHIN THE HANSEL TESTBED: FRAMEWORK OVERVIEW}

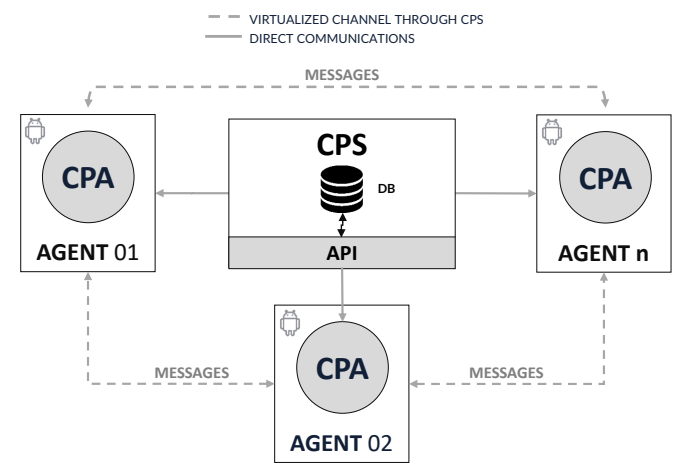

Fig. 6: Client-server approach applied to a star topology for CP PoC.

The high-level infrastructure on which the $\mathrm{PoC}$ is based has a star topology that relies on a center node (e.g. remote server) implementing the Collaborative Positioning Service (CPS). The CPS is at the same time a buffer which collects raw GNSS measurements and a virtual agent-to-agent channel established between registered application instances (Collaborative Positioning Application (CPA)), as shown in Figure 6. A distributed framework over general-purpose network connectivity (i.e. 4G/LTE) has been exploited in this implementation. Such topology ensures a centralized data collection and independent and distributed PVT computation. In this way, the computational load is expected by the agent nodes, while the central node is in charge of managing the data distribution and any possible policy for data dispatching. In the following, a detailed discussion is provided about the software implementation of the PoC.

\section{A. Collaborative Positioning Service}

The CPS provides registration and authentication facilities to the agents through the methods of a dedicated API, and it foresees a database, referred hereafter as $\mathrm{db}$, for the buffering of raw measurements posted by the registered users. The CPS can handle an arbitrary number of users according to the computational capability of the hosting server and to the network performance. However, these aspects fall outside the scope of this article.

1) Raw Measurements Database: The $\mathrm{db}$ is intended as a dynamic, centralized data buffer oriented to the agile exchange among the registered agents. It was implemented following a document data model paradigm [53]. The data are stored in collections of files which are dynamically allocated whenever an agent performs the registration to the CPS, and whenever it shares new measurements through the service. When a new agent subscribes to the CPS, a new entry is created, uniquely indexed, and continuously updated by the remote CPA running on the relative mobile device. All the data of interest for localization such as raw measurements, coarse position estimates, timestamps and cooperative parameters provided by the agents are hence stored in an instance of the database running for the CPS.

2) Cooperative Positioning System API: As shown in Figure 6 , the CPS API is conceived as a unique entry point of the framework for the mobile agents. It is implemented through a Python script and it exploits the following actions:

- Agent registration: It is provided by the interaction between CPS and CPA instances running on the smartphones, and registered users list can be monitored runtime through the methods provided by the API.

- Raw measurements download and monitoring: UDP sockets are provided by the CPS to manage the upstream and downstream of data among the agents. Such data are encapsulated in UDP messages called Collaborative Raw Message (CRM) which can be downloaded from the CPS to perform $\mathrm{CP}$.

- Housekeeping of the database content: The CPS provides a storage-efficient housekeeping routine in order to delete all the outdated raw measurements collections. Through the methods included in the API, it allows to define a refresh time for the deletion of outdated records and to download all the records stored by the db for further analysis and post-processing.

\section{B. Collaborative Positioning Application - CPA}

The CPA constitutes the core entity of the collaborative framework. It allows an agent to interact with other agents through the CPS. The CPA is a multi-thread application which foresees a main script managing a set of sub-threads running in parallel. They can be seen as microservices producing and consuming local and external measurements. Independent instances of the CPA are expected to interact through the CPS by means of the service API described so far in order to benefit from the collaboration. According to the overall functional scheme of the CPA presented in Figure 7, the following components have been implemented. 


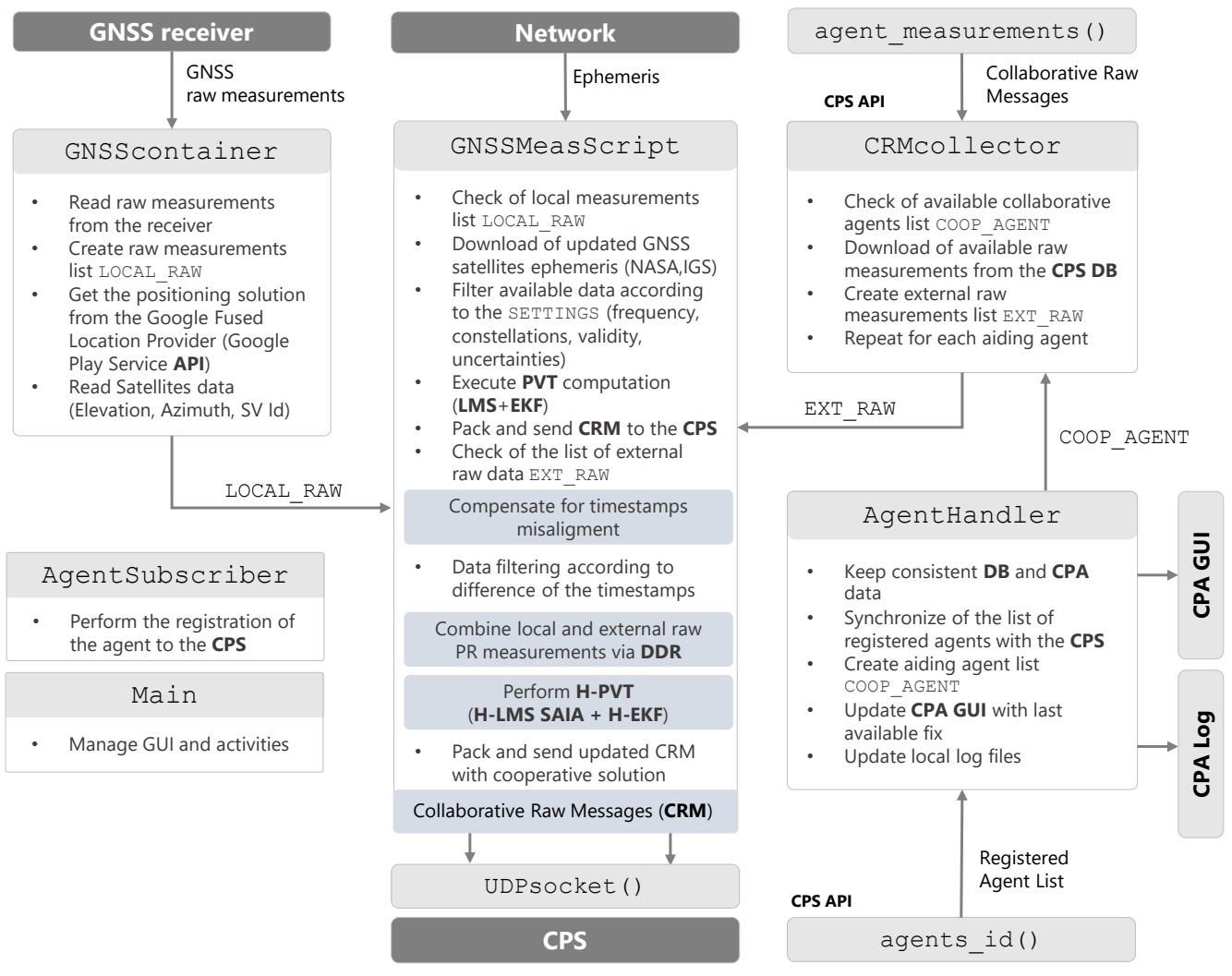

Fig. 7: Full CPA functional scheme showing all the threads and the transferred data structures.

1) Main activity: It is the entry point of the CPA as it is in charge of managing the Graphic User Interface (GUI) and the life cycle of the whole application. It runs specific threads according to the incoming commands from the users.

2) Agent Subscriber: The registration to the CPS is compulsory to join the collaborative framework.

Given available connectivity among CPS and CPA instances, after the startup of the application, the following steps are executed

- The CPA sends a request for registration to CPS

- The CPS acts to avoid concurrent requests and duplicated identifiers by randomizing its response time.

- The CPA receives an authentication token to guarantee authenticated client-server communication along with a unique agent identifier for the identification of active users of the CPS multi-agent system.

Once the subscription is accomplished, the four main parallel threads are operated by the CPA in order to allow to the new agent to broadcast and download raw measurements: the GNSSContainer, the CRMcollector, the AgentHandler and the GNSSMeasScript.

3) GNSS Container: The GNSScontainer is in charge of reading raw measurements from the onboard GNSS receiver. It manages the creation of a list named LOCAL_RAW including raw pseudorange and Doppler measurements obtained at the current epoch. Visible and trackable satellites are also identified as well as their relative position (azimuth, elevation) w.r.t. to the agent location. All the data along with their unique identifiers (e.g. G07, E21, R04) are also logged to know current visibility conditions through a specific callback. In parallel, it collects the last available fix through the onboard GNSS/INS accurate solution to be used as a ground-truth for the GNSS standalone and collaborative positioning solutions. The list LOCAL_RAW is eventually sent to GNSSMeasScript to be processed within the PVT algorithm.

4) Agent Handler: The AgentHandler thread is in charge of keeping information between CPA and the $\mathrm{db}$ coherent and updated. It is the only thread interacting with the CPS API during the operational life of the service. This implementation ensures strong independence among the components aiming at guaranteeing effortless portability of the framework.

5) CRMcollector: If the GNSScontainer can be seen as the interface between the CPA and the GNSS receiver, the CRMcollector is the interface between the CPA and the network of aiding agents. It receives the updated list of potential aiding agents, COOP_AGENT from the AgentHandler and it is in charge of retrieving the available raw measurements stored in the db by invoking the method agent_measurements. The download of such data is performed through the aforementioned UDP socket. The early version of the CPA foresees a serial CRMcollector which sequentially creates a list of raw measurements and GNSS standalone estimates from each 
aiding agent, named EXT_RAW. The list EXT_RAW is then sent to the GNSSMeasScript to perform the core collaborative task.

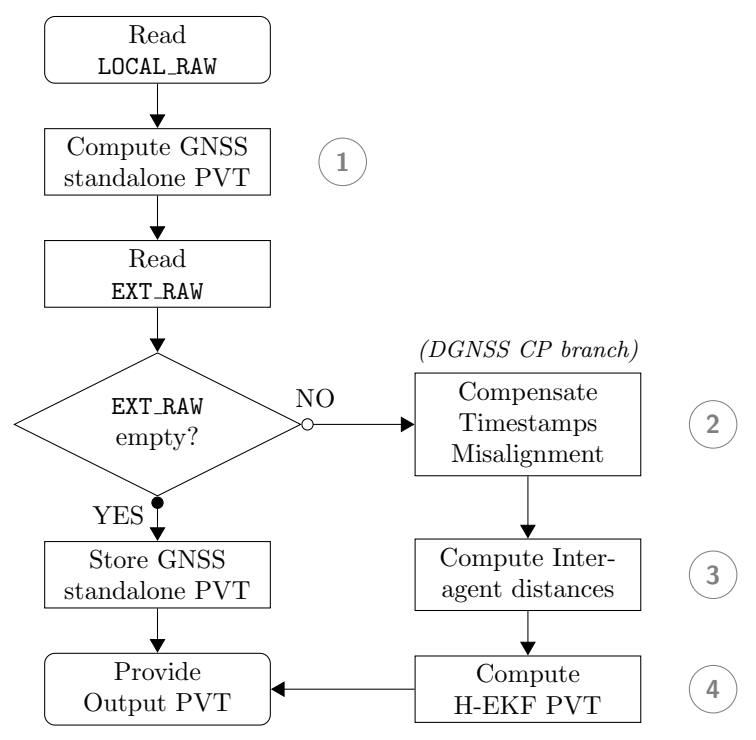

Fig. 8: High-level hybrid positioning flow chart of the GNSSMeasScript.

6) GNSSMeasScript: It is responsible for the execution of the fundamental steps listed in Section III and summarized in the flow-chart of Figure 8.

a) Coarse Position and Time Estimation (1): The raw measurements obtained from the GNSScontainer are processed to compute a GNSS standalone positioning solution, referred to as coarse PVT. Such a solution can be computed both through a H-WLS or through a Hybrid Extended Kalman Filter (H-EKF), as described in Section III-A. The timestamps provided by the GNSS receivers are used to label each set of raw measurements and related fix included in the CRM with the field epoch.

b) Compensation of time-misalignment of local and downloaded measurements (2): This step exploits the local raw Doppler shift measurements provided by the API, to compensate for the time misalignment among the measurements in LOCAL_RAW and the ones included in EXT_RAW, as discussed in Section III-C1 according to (11).

c) DGNSS inter-agent distance estimation (3): Timealigned measurements are combined through WDD to determine the inter-agent distances among the target agent and the aiding ones, as presented in III-C2, according to (16).

d) Hybridized PVT estimation (4): An initial $\mathrm{H}$ WLS/WLS solution is mandatory for the initialization of the main navigation filter and it is forced every $N$ fixes to avoid unexpected drifting phenomena due to unmodelled threats, according to the EKF architecture discussed in Section III-D. For the same reason H-EKF/EKF can be disabled when it is not suitable for the positioning computation in order to reduce the correlation of the positioning solutions along the time (i.e. static scenario). Functional blocks 2, 3, and 4 in the scheme of Figure 8 can be replaced by improved algorithms to accomplish each specific task, thus implementing different $\hat{d}_{a b}$ estimation techniques, improved synchronization, and hybridization solutions.

\section{EXPERIMENTAL SETUP}

All the tests were performed addressing different static and kinematic scenarios to verify and assess feasibility and effectiveness of the proposed $\mathrm{CP}$ through the interaction of two selected smartphones running independent instances of the CPA. GNSS capabilities of both the smartphones were limited to the single constellation (GPS) and single frequency (L1) positioning computation by further limiting the number of visible satellites to emulate poor sky visibility, if necessary. The devices were handled by two operators walking or driving vehicles according to the designed test paths. A weighting factor applied to the measurement covariance matrix, $\mathbf{R}_{k}$, was used as a EKF design parameter to control the impact of the GNSS measurements w.r.t. the linear model forward prediction in the EKF position estimation algorithm for both collaborative and standalone solutions.

\section{A. Scenarios}

The preliminary testing phase addressed the peer-to-peer cooperation with ultra-short, short, and moderate baseline length. The two smartphones, referred hereafter as agent SMO1 and agent SM02, were indeed configured in order to continuously exchange GNSS data by exploiting 4G/LTE data connectivity, and a general-purpose Amazon Web Service (AWS) server hosting the CPS on a server physically located in Dublin, Ireland. Being independent of the behavior of the other agent, each smartphone attempted to improve its positioning accuracy by exploiting shared GNSS raw measurements and coarse fixes shared by the other agent, thus acting simultaneously as aiding and aided agent.

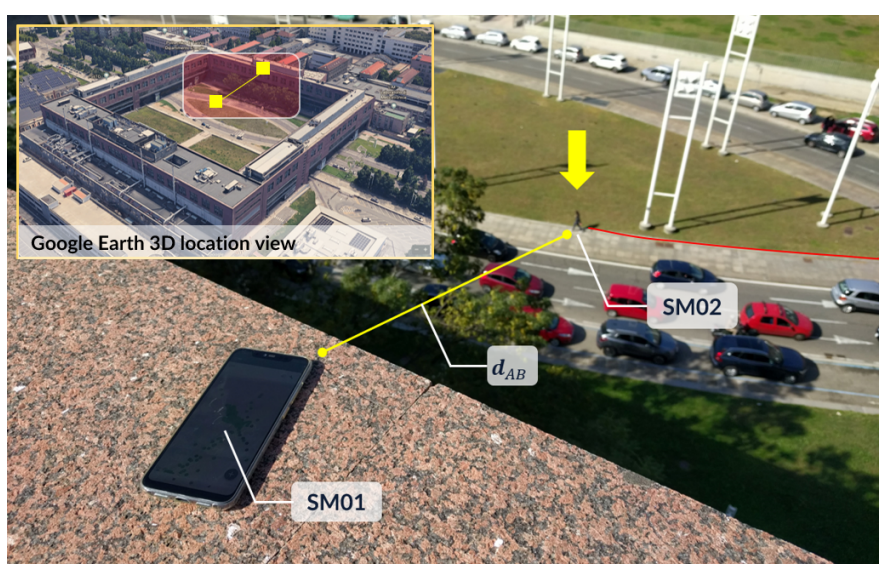

Fig. 9: Example of pedestrian urban scenario with anchored variable baseline vector (experiment C-01). Poltiecnico Campus, Turin, Italy.

Each device was hence able to independently compute collaborative inter-agent measurements by combining external and local observables. Such an operational mode was helpful to observe both benefits and disadvantages of the proposed integration by identifying timespans in which CP was actually 
effective and other timespans in which it induced degradation of the positioning solution. In fact, an actual implementation is expected to inhibit $\mathrm{CP}$ when a-priori conditions suggest a potentially degraded performance, as discussed about the fundamental limits of DGNSS CP [48]. Similar (near-zero inter-agent distance) or different paths were travelled by the operators holding the devices and different effects were observed accordingly for each smartphone under test, as it will be reported in Section VI. All the positioning data were sent to the CPS and logged locally in form of Earth Centered Earth Fixed (ECEF) and Latitude, Longitude, Altitude (LLA) positioning solution. Both cooperative and standalone solutions were then compared to the reference location foreseen by the on-board GNSS/INS accurate solution, according to the metric defined in Section V-B.

\section{B. Performance metrics}

A set of performance metrics are defined hereafter to evaluate the benefits of the proposed DGNSS CP within the on-field tests operated through the PoC.

a) availability: , $\mathcal{A}_{\mathrm{CP}}$ : It measures the availability of the cooperative service for a given agent by assessing the possibility of computing a hybrid positioning solution. It is an indirect indicator of the network status and the overall availability of raw measurements provided by registered aiding agents. It formally counts the ratio between the number of CP fixes, $N_{\mathrm{CP}}$, and the overall number of available GNSS standalone fixes, $N_{\mathrm{SA}}$

$$
\mathcal{A}_{\mathrm{CP}}=\frac{N_{\mathrm{CP}}}{N_{\mathrm{SA}}} 100 .
$$

Even considering a full availability of the service, $\mathcal{A}_{\mathrm{CP}}=$ 100 , as known from preliminary simulation campaign DGNSS $\mathrm{CP}$ could be profitable or unprofitable according to the quality of the collaborative measurements and the relative geometry of visible satellites and aiding agents [48]. To assess the advantages of the proposed technique, the following key metrics have been defined to quantify the accuracy of the positioning solutions.

b) Positioning Error: : it can be evaluated as the Euclidean distance of the estimated position from the ground truth in 3-D (spatial error)

$$
\xi_{3-\mathrm{D}, k}=\sqrt{\left(\Delta E_{k}^{2}+\Delta N_{k}^{2}+\Delta U_{k}^{2}\right)}
$$

and in 2-D (horizontal error)

$$
\xi_{2-\mathrm{D}, k}=\sqrt{\left(\Delta E_{k}^{2}+\Delta N_{k}^{2}\right)}
$$

where the squared terms in (22) and (21) are the difference of each component in a East-North-Up (ENU) reference frame [54], [55]. It is helpful to recall that ENU coordinates are obtained from linear transformation of ECEF coordinates so that (21) can be equivalently computed in ECEF. The ground truth is provided in this case by the on-board GNSS/INS solution according to its higher accuracy due to inertial sensors integration. For the sake of simplicity, the errors will be always referred to as $\xi_{\mathrm{SA}}$ and $\xi_{\mathrm{CP}}$ for standalone and $\mathrm{CP}$ solutions, respectively. Separate metrics for 3-D and 2-D will be then considered in Section VI. c) $C P$ profitability: $(2-\mathrm{D}, 3-\mathrm{D}), \mathcal{P}_{\mathrm{CP}}(\%)$ : It indicates the percentage of $\mathrm{CP}$ fixes in which $\mathrm{CP}$ error is lower than standalone positioning error (i.e. GPS-only), formally

$$
\mathcal{P}_{\mathrm{CP}}(\%)=\frac{\sum_{k=1}^{k=N_{\mathrm{CP}}} p_{k}}{N_{\mathrm{CP}}}
$$

where $p_{k}$ assumes boolean values according to the following conditions

$$
\begin{cases}p_{k}=0 & \xi_{\mathrm{SA}, k}-\xi_{\mathrm{CP}, k}<-T_{H} \\ p_{k}=1 & \xi_{\mathrm{SA}, k}-\xi_{\mathrm{CP}, k}>+T_{H}\end{cases}
$$

where $T_{H}$ is a hysteresis threshold used to perform a conservative classification of profitable/unprofitable epochs.

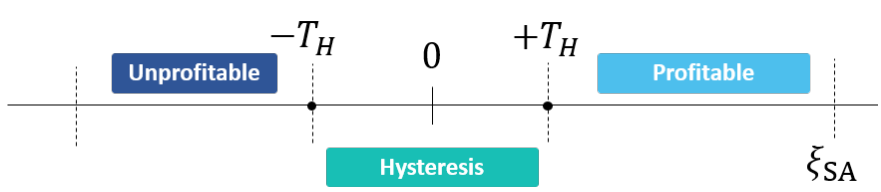

Fig. 10: Classification of profitability according to the hysteresis threshold $T_{H}$.

d) CP hysteresis: , $\mathcal{H}_{\mathrm{CP}}(\%)$ : It counts all the epochs $t_{k}=$ $k$ in which $\left|\xi_{\mathrm{SA}, k}-\xi_{\mathrm{CP}, k}\right|<T_{H}$, as depicted in Figure 10.

The hysteresis identifies a region of non-significant improvement/worsening of the estimation accuracy. It is worth remarking that this percentage highlights epochs in which $\mathrm{CP}$ does not guarantee benefits and it should be inhibited.

e) Mean CP error : (2-D,3-D), $\mathcal{E}_{\mathrm{CP}}(\mathrm{m})$ : It computes the mean positioning error of $\mathrm{CP}$ w.r.t. on-board accurate navigation solution over the time epochs in which $\mathrm{CP}$ is profitable

$$
\mathcal{E}_{\mathrm{CP}}=\frac{1}{W} \sum_{w=1}^{W} \xi_{k}
$$

where $W=\sum p_{k}$ counts the overall amount of profitable epochs, formally $\forall k \mid p_{k}=1$.

f) Mean SA positioning error: (2-D,3-D), $\mathcal{E}_{\mathrm{SA}}(\mathrm{m})$ : It is equivalent to $\mathcal{E}_{\mathrm{CP}}$ evaluated for the GNSS standalone solution

g) $C P$ accuracy mean improvement: (2-D,3-D), $\mathcal{I}_{\mathrm{CP}}$ : It provides the percentage of the improvement in accuracy guaranteed epoch-by-epoch by the $\mathrm{CP}$ when the cooperation is profitable. It can be also seen as GNSS-relative error reduction, formally

$$
\mathcal{I}_{\mathrm{CP}}(\%)=\frac{1}{W} \sum_{j=1}^{W} 1-\frac{\xi_{\mathrm{CP}}\left(t_{j}\right)}{\xi_{\mathrm{SA}}\left(t_{j}\right)}
$$

$\mathcal{I}_{\mathrm{CP}}$ immediately provides an indicator of the accuracy improvement of the hybrid solution w.r.t. the GNSS standalone fix. When $\mathcal{I}_{\mathrm{CP}} \simeq 100 \%$, the hybrid solution shows an appreciable match with the fix provided by the on-board GNSS/INS navigation solution, while when $\mathcal{I}$ approaches 0 , the hybrid fix fairly matches with the GNSS standalone solution.

All the accuracy metrics in (25),(24) and (23) can be computed for 2-D and 3-D by replacing the corresponding error metrics (22) and (21), in the respective equations. 


\section{RESULTS}

A set of experiments have been chosen to present the relevant results observed during an early test campaign. Extended results and discussion are shown for a single zero-baseline assessment test while quantitative data obtained through a full test campaign are summarized and discussed by analyzing the overall behaviour of the performance metrics and commenting Figures 12a, 12b, 13 and 14 accordingly.

\section{A. Sample Experiment f-01: Zero-baseline}

This test was chosen to assess the feasibility of the proposed CPA/CPS architecture dealing with raw measurements coming from identical devices located together in the same reference location.

- Description: SM01 and SM02 are statically co-located at centre of a parking lot in the Politecnico Campus (Turin, Italy).

- Visibility: urban environment (buildings, trees) with good portion of open sky.

- Constraints: forced to poor visibility using the best 5 GPS satellite in view.

- EKF parameters: multiplicative measurements covariance weight set to 20 .

- Server location (CPS host): AWS eu-west-1 Europe (Dublin, Ireland).

According to the profitability results shown in Figure 11a, it is worth noticing that $\mathrm{SMO} 2$ benefits from $\mathrm{CP}$ for more than $40 \%$ of the data collection. It would be reasonable to assume that SMO1 did not benefit from the aids of SMO2 during the same epochs.

In light of this, the plot presented in Figure 11b highlights such a fundamental aspects of this pairwise collaboration: by looking at time $t_{k}$ from $40 \mathrm{~s}$ to $140 \mathrm{~s}$ we notice indeed that the collaboration is more profitable for SMO1 than SMO2. This behavior is attributed to the overall good quality of the raw measurements and consequently to the goodness of the coarse fix of SM02. It is indeed worthy to recall that the coarse position estimate of the aiding agents is used as a reference for the tight-integration of the inter-agent distance, as detailed in the tight-integration algorithm defined in Section III. The quantitative comparison of the mean positioning error shown in the correspondent plot of Figure 15 confirms that, during profitable epochs, SMO1 obtained a negligible benefit from cooperation while the opposite was observed for SM02.

\section{B. Test Campaign: Summary of the results}

A further assessment for the feasibility of the paradigm is given through the analysis of the CDFs of the positioning errors, provided in Figure 16. Different from the previous analysis, the plots depict the overall error that affect the positioning solutions over the whole test duration. A comparison is shown between standalone and cooperative solution and a negligible improvement can be noticed for low dynamics scenarios such as for the experiments $c-01$ and $d-01$. On the contrary, a higher advantage is provided on average for high relative dynamics as for the sample experiment $d-02$,
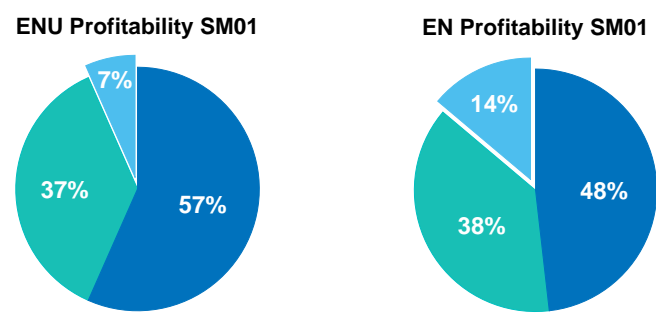

ENU Profitability SM02
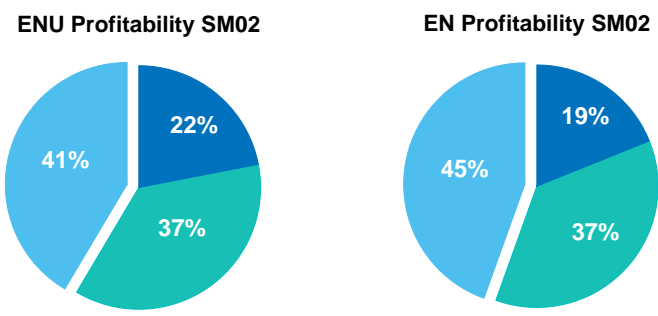

Profitable

Invariant Unprofitable

(a)

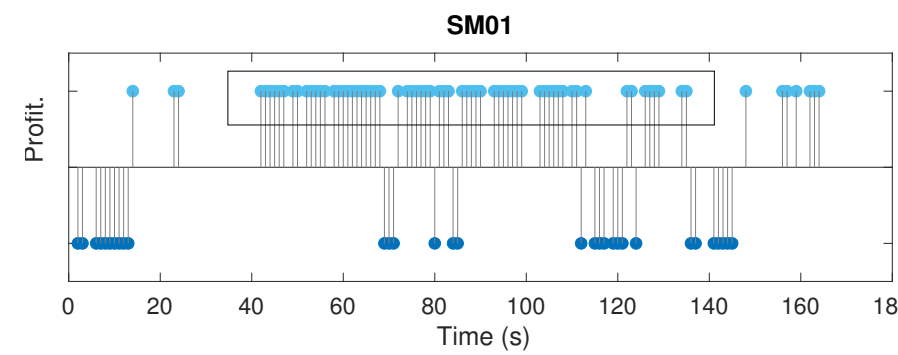

SM02

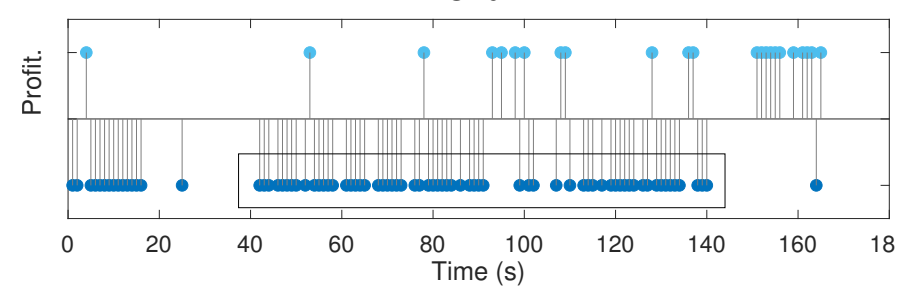

(b)

Fig. 11: Sample results from the experiment f-01: (a) Pie charts of CP profitability for the two smartphone, (b) binary time series of profitability/unprofitability.

in which the cooperation was established within bicyclepedestrian pair (bottom-left quadrant). A different case is instead shown in the upper-right quadrant for the experiment $f-1$ in which a clear aiding-aided configuration would be advisable as $\mathrm{SMO} 1 \rightarrow \mathrm{SMO}$. This finding is clearer in static experiments during which two identical devices surprisingly experienced different navigation conditions over the whole timespan.

1) Availability: The average availability of the $\mathrm{CP}$ across all the experiments, shown in Figure 12a, was about $86.94 \%$ and $81.23 \%$ of the whole test duration for SMO1 and SM02 respectively. If we do not consider the set of experiments g-0x for which the device SM02 experienced unexpected 


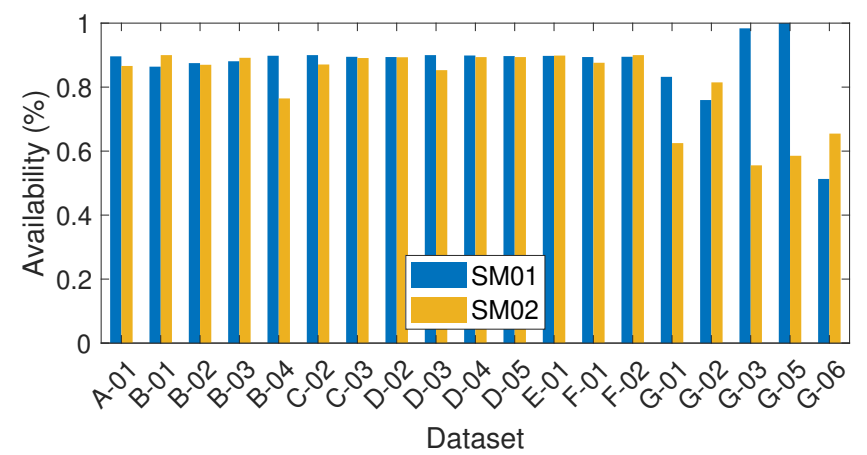

(a)

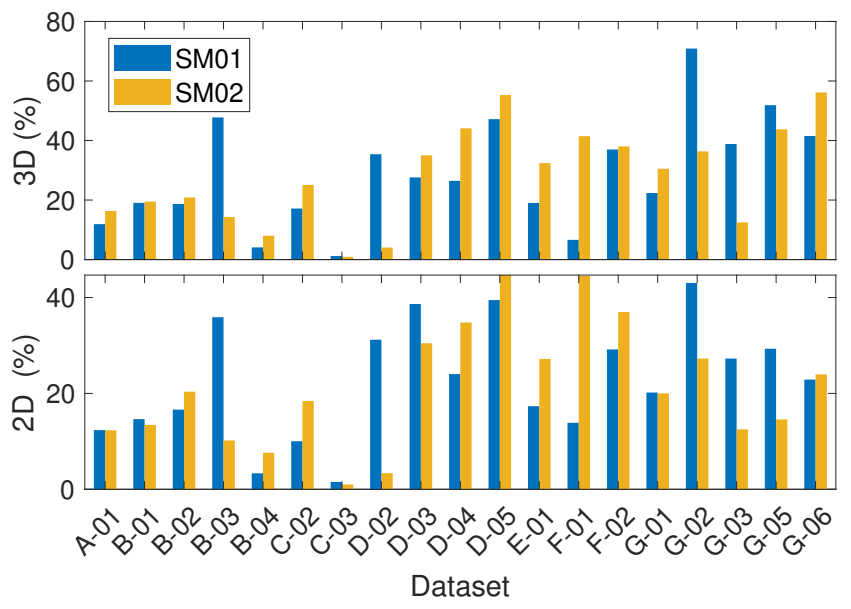

(b)

Fig. 12: (a) availability of the collaborative measurements from CPS during the experimental timespan, (b) 3D and 2D Profitability of the CP paradigm from CPA log files.

connectivity issues, the availability can be considered above the $87 \%$ for a pairwise $\mathrm{CP}$ accomplished through the specific $4 \mathrm{G} / \mathrm{LTE}$ connectivity used in the tests. Let us remark that this metric is tightly related to the network congestion and latency that can be considerably different according to the infrastructure and topology; The measurement combination was indeed excluded if the difference between raw data timestamps of the devices exceeded a given threshold (i.e. $1 \mathrm{~s}$ ), according to the PoC implementation described in Section IV. Although it is fundamental to rely on low-latency communication networks to limit the amount of discarded data, a detailed analysis of the network infrastructure and of possible Quality of Service (QoS) policies that could guarantee priority to the CRMs are out of the scope of this work. Furthermore, the presented paradigm can be considered to be implemented through $5 \mathrm{G}$ New Radio (NR) URLLC or even device-to-device technologies (e.g. Direct Short Range Communication (DSRC)) to avoid bottlenecks experienced at the server-side.

2) Expected improvement in real scenarios: By averaging the results reported in Figure 14, the mean 2-D accuracy improvement provided by the $\mathrm{CP}$ across al the experiments is about $40.7 \%$ for $\mathrm{SMO} 1$ and about $43.5 \%$ for SMO2. By considering the different conditions of each experiment, this result suggests an expected value of the accuracy improvement (in the case of profitable conditions) for a single collaborative contribution in poor satellite visibility and mild-urban environment.

\section{CONCLUSIONS}

In this paper, a validation through the implementation of a PoC is presented for a cooperative, sensor-less, and GNSSonly positioning technique able to cope with the increasing requirement of precision and accuracy for GNSS positioning in harsh scenarios. By exploiting the general-purpose mobile 4G/LTE network and raw-measurements-enabled smartphones, the developed PoC demonstrated the feasibility of a cooperative positioning framework that allows the near-realtime exchange of raw GNSS measurements among consumer smartphones, the estimation of the inter-agent distance, and the evaluation of a hybrid PVT solution with potential highest accuracy w.r.t. the GNSS standalone solution. The use of a remote server hosting the back-end service demonstrated that the network latency affecting the data transmissions plays a marginal role in limiting the overall availability of such auxiliary measurements, which is on average above the $80 \%$ of the duration of the tests.

It has been shown that when collaborative inter-agent measurements were available and profitable in the pairwise configuration under test, the average improvement w.r.t. to the standalone GNSS solution overcame the $40 \%$, both in bi-dimensional and three-dimensional reference frames. Further improvements can be expected by exploiting multiconstellation and multi-frequency distance computation to improve the reliability against multipath [56], and by implementing a selection algorithm of the agents to be combined according to the geometry of the relative positions of satellites and collaborating agents. Future works will address protocol definition and scalability issues when more than two devices are considered cooperating.

\section{ACKNOWLEDGEMENTS}

The Proof of Concept presented in this paper was designed, developed, and tested in the context of HANSEL ${ }^{1}$ [23], an ESA-funded Technology Research Programme (TRP) activity. The authors would like to acknowledge ESA and all the partners of the HANSEL consortium, especially Rokubun team for leading and supporting the design, development, and integration of this PoC and the whole HANSEL testbed.

\section{REFERENCES}

[1] European GNSS Agency (GSA), "Market report issue 6," European Global Navigation Satellite Systems Agency, Oct 2019.

[2] M. Zhu, X. Liu, F. Tang, M. Qiu, R. Shen, W. Shu, and M. Wu, "Public vehicles for future urban transportation," IEEE Transactions on Intelligent Transportation Systems, vol. 17, no. 12, pp. 3344-3353, Dec. 2016.

[3] M. Cao, L. Zheng, W. Jia, and X. Liu, "Fast monocular visual odometry for augmented reality on smartphones," IEEE Consumer Electronics Magazine, pp. 1-1, 2020.

${ }^{1}$ ESA contract number 4000126230/18/NL/CRS 


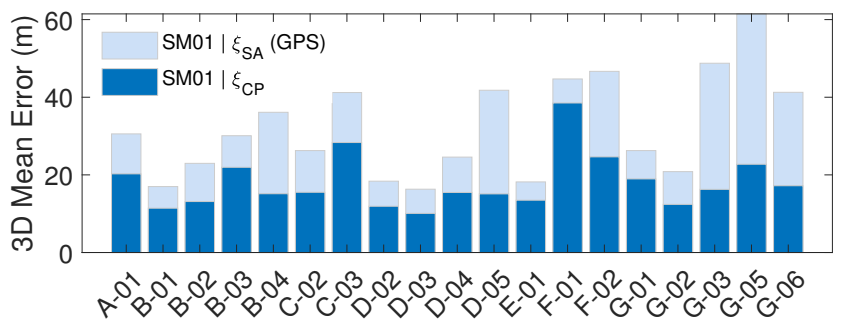

(a)

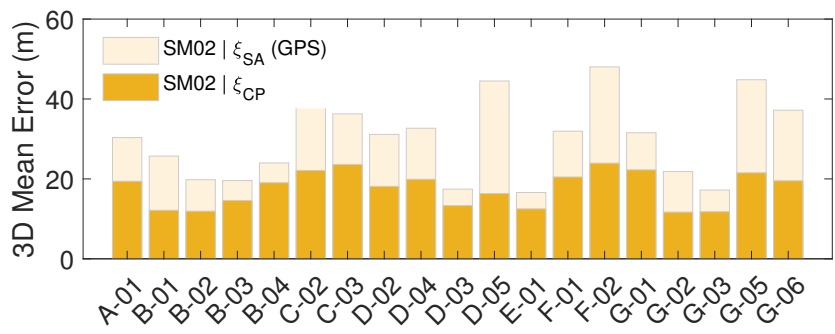

(c)

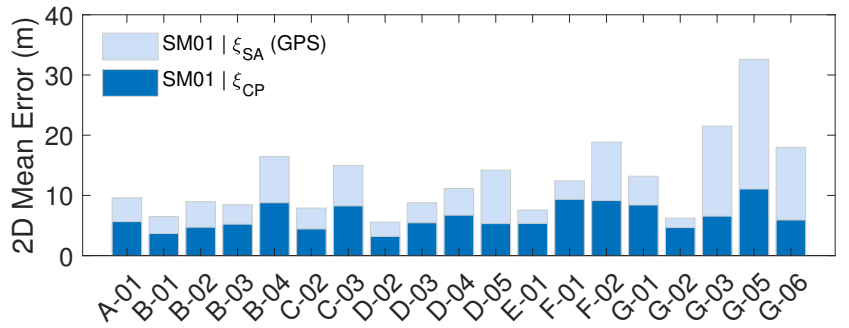

(b)

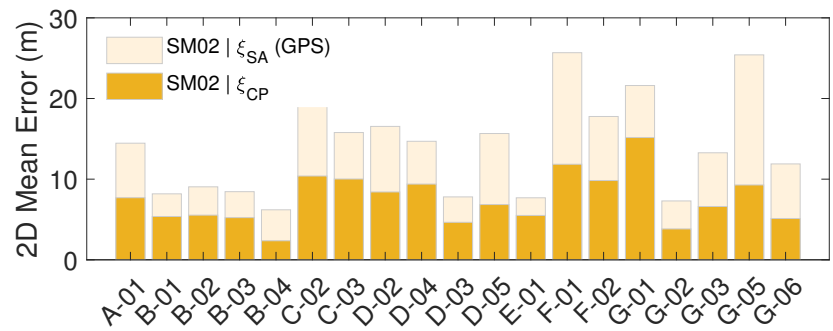

(d)

Fig. 13: Comparison between GNSS standalone and CP mean positioning errors.

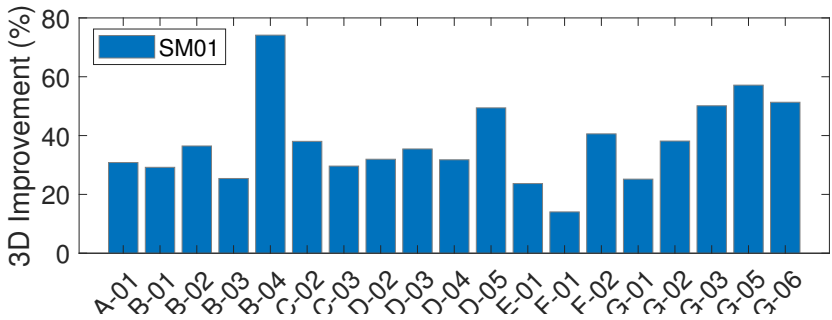

(a)

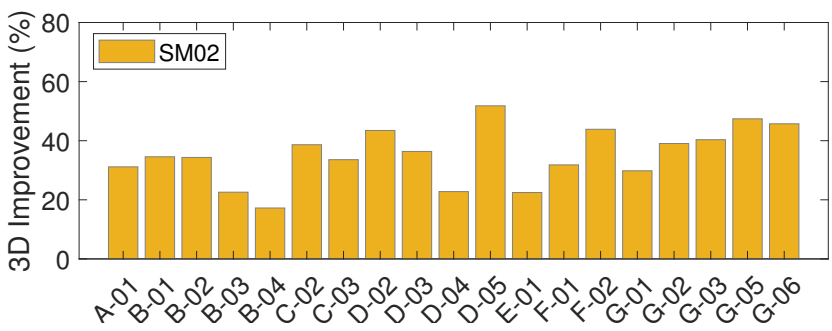

(c)

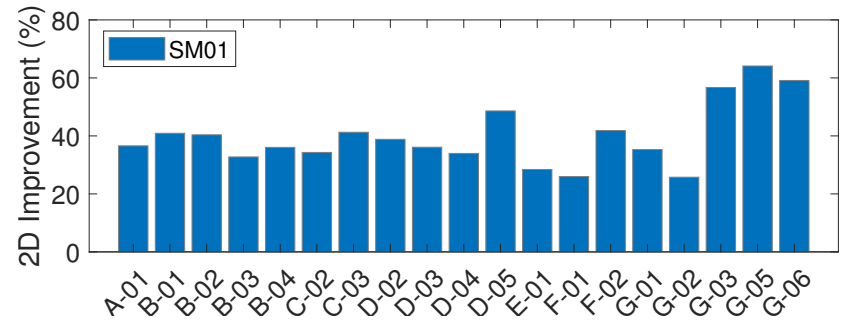

(b)

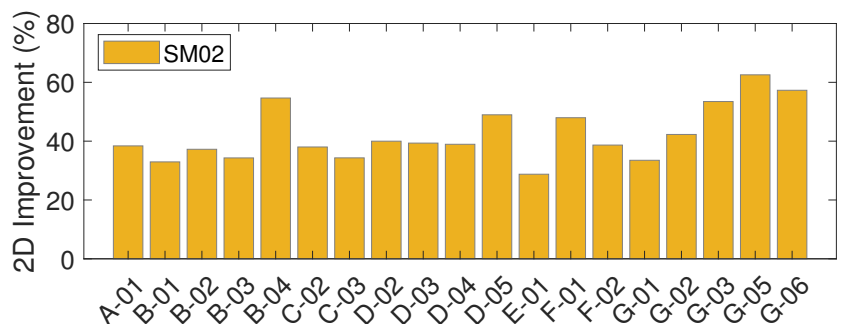

(d)

Fig. 14: Mean accuracy improvement achieved through CPA/CPS interaction in the different tests.

[4] C. H. Liu, X. Ma, X. Gao, and J. Tang, "Distributed energy-efficient multi-uav navigation for long-term communication coverage by deep reinforcement learning," IEEE Transactions on Mobile Computing, vol. 19, no. 6, pp. 1274-1285, Mar. 2020.

[5] M. S. Grewal, L. R. Weill, and A. P. Andrews, Global positioning systems, inertial navigation, and integration. John Wiley \& Sons, 2007.

[6] I. Skog and P. Handel, "In-car positioning and navigation technologies: A survey," IEEE Transactions on Intelligent Transportation Systems, vol. 10, no. 1, pp. 4-21, Mar. 2009.

[7] G. Falco, M. Pini, and G. Marucco, "Loose and tight GNSS/INS integrations: Comparison of performance assessed in real urban scenarios," Sensors, vol. 17, no. 2, 2017. [Online]. Available: http://www.mdpi.com/1424-8220/17/2/255

[8] C. Cristodaro, F. Dovis, G. Falco, and M. Pini, "GNSS receiver performance in urban environment: Challenges and test approaches for automotive applications," in 2017 International Conference of Electrical and Electronic Technologies for Automotive, June 2017, pp. 1-6.
[9] N. Zhu, J. Marais, D. Bétaille, and M. Berbineau, "GNSS position integrity in urban environments: A review of literature," IEEE Transactions on Intelligent Transportation Systems, vol. 19, no. 9, pp. 2762-2778, Sep. 2018.

[10] C. Cristodaro, "Advanced integration of GNSS and external sensors for autonomous mobility applications, doctoral thesis," 2019.

[11] Y. Zhuang and N. El-Sheimy, "Tightly-coupled integration of WiFi and MEMS sensors on handheld devices for indoor pedestrian navigation," IEEE Sensors Journal, vol. 16, no. 1, pp. 224-234, Jan. 2016.

[12] A. Sheta, A. Mohsen, B. Sheta, and M. Hassan, "Improved localization for Android smartphones based on integration of raw GNSS measurements and IMU sensors," in 2018 International Conference on Computer and Applications (ICCA), Aug. 2018, pp. 297-302.

[13] G. Rodriguez, F. Casado, R. Iglesias, C. Regueiro, and A. Nieto, "Robust step counting for inertial navigation with mobile phones," Sensors, vol. 18, no. 9, p. 3157, Sep. 2018.

[14] M. L. Cherif, J. Leclère et al., "Loosely coupled GPS/INS integration 


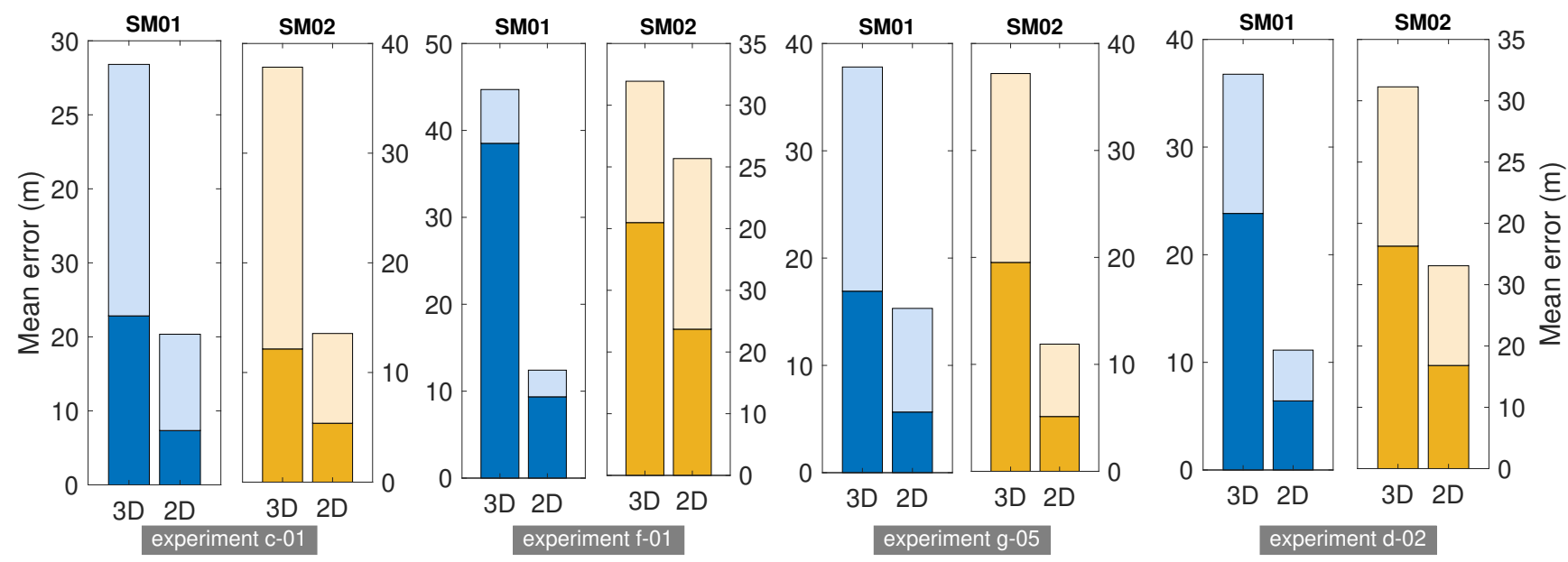

Fig. 15: Collection of mean errors comparisons for the sample datasets selected among the experiments of the test campaign.
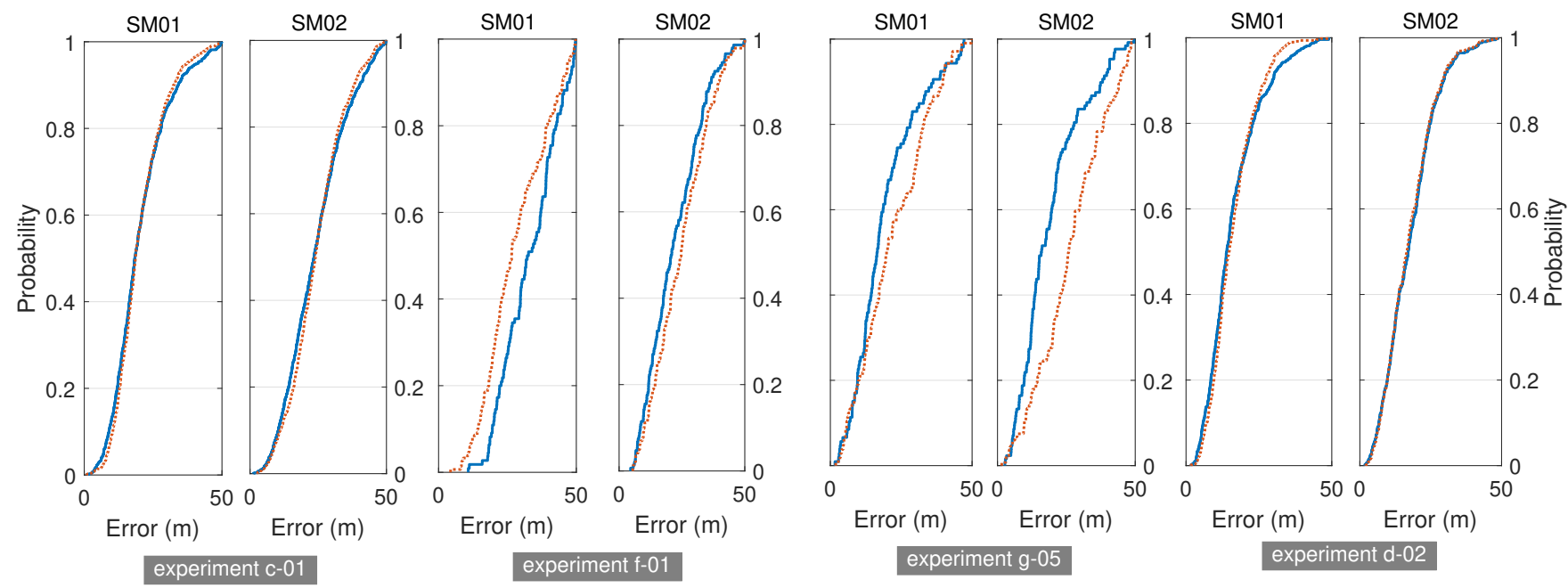

Fig. 16: Collection of Cumulative Density Function (CDF)s for the sample datasets selected among the experiments of the test campaign.

with snap to road for low-cost land vehicle navigation: EKF-STR for low-cost applications," in 2018 IEEE/ION Position, Location and Navigation Symposium (PLANS). IEEE, 2018, pp. 275-282.

[15] J. Dong, M. Noreikis, Y. Xiao, and A. Ylä-Jääski, "Vinav: A visionbased indoor navigation system for smartphones," IEEE Transactions on Mobile Computing, vol. 18, no. 6, pp. 1461-1475, Jul. 2019.

[16] K. Shamaei, J. Khalife, and Z. M. Kassas, "Exploiting LTE signals for navigation: Theory to implementation," IEEE Transactions on Wireless Communications, vol. 17, no. 4, pp. 2173-2189, Apr. 2018.

[17] J. Khalife and Z. M. Kassas, "Navigation with cellular CDMA signals-part II: Performance analysis and experimental results," IEEE Transactions on Signal Processing, vol. 66, no. 8, pp. 2204-2218, Apr. 2018.

[18] Y. Zhou, C. L. Law, Y. L. Guan, and F. Chin, "Indoor elliptical localization based on asynchronous UWB range measurement," IEEE Transactions on Instrumentation and Measurement, vol. 60, no. 1, pp. 248-257, Jan. 2011.

[19] M. Rohani, D. Gingras, and D. Gruyer, "Dynamic base station DGPS for cooperative vehicle localization," in 2014 International Conference on Connected Vehicles and Expo (ICCVE), Nov. 2014, pp. 781-785.

[20] J. N. Gross, Y. Gu, and M. B. Rhudy, "Robust UAV relative navigation with DGPS, INS, and peer-to-peer radio ranging," IEEE Transactions on Automation Science and Engineering, vol. 12, no. 3, pp. 935-944, Jul. 2015.

[21] A. Minetto, "GNSS-only collaborative positioning methods for net- worked receivers, doctoral thesis," Ph.D. dissertation, Politecnico di Torino, 2020.

[22] A. Minetto, A. Nardin, and F. Dovis, "GNSS-only collaborative positioning among connected vehicles," in Proceedings of the 1st ACM MobiHoc Workshop on Technologies, mOdels, and Protocols for Cooperative Connected Cars, ser. TOP-Cars '19. New York, NY, USA: ACM, 2019, pp. 37-42. [Online]. Available: http://doi.acm.org/10.1145/3331054.3331552

[23] A. Minetto, F. Dovis, A. Vesco, M. Garcia-Fernandez, 1. López-Cruces, J. L. Trigo, M. Molina, A. Pérez-Conesa, J. Gáñez-Fernández, G. Seco-Granados, and J. A. López-Salcedo, "A testbed for gnss-based positioning and navigation technologies in smart cities: The hansel project," Smart Cities, vol. 3, no. 4, pp. 1219-1241, 2020. [Online]. Available: https://www.mdpi.com/2624-6511/3/4/60

[24] D. Yang, F. Zhao, K. Liu, H. B. Lim, E. Frazzoli, and D. Rus, "A GPS pseudorange based cooperative vehicular distance measurement technique," in 2012 IEEE 75th Vehicular Technology Conference (VTC Spring), May 2012, pp. 1-5.

[25] M. Tahir, S. S. Afzal, M. S. Chughtai, and K. Ali, "On the accuracy of inter-vehicular range measurements using GNSS observables in a cooperative framework," IEEE Transactions on Intelligent Transportation Systems, pp. 1-10, Jun. 2018.

[26] A. Minetto, C. Cristodaro, and F. Dovis, "A collaborative method for positioning based on GNSS inter agent range estimation," in 2017 25th European Signal Processing Conference (EUSIPCO), August 2017, pp. 2714-2718. 
[27] N. Alam, A. Tabatabaei Balaei, and A. G. Dempster, "Relative positioning enhancement in VANETs: A tight integration approach," IEEE Transactions on Intelligent Transportation Systems, vol. 14, no. 1, pp. 47-55, Mar. 2013.

[28] — - "A DSRC doppler-based cooperative positioning enhancement for vehicular networks with GPS availability," IEEE Transactions on Vehicular Technology, vol. 60, no. 9, pp. 4462-4470, Nov. 2011.

[29] A. Minetto, A. Nardin, and F. Dovis, "Tight integration of GNSS measurements and GNSS-based collaborative virtual ranging," in 31st International Technical Meeting of the Satellite Division of The INStitute of Navigation (ION GNSS+ 2018), September 2018, pp. 2399-2413.

[30] K. A. Redmill, T. Kitajima, and U. Ozguner, "DGPS/INS integrated positioning for control of automated vehicle," in ITSC 2001. 2001 IEEE Intelligent Transportation Systems. Proceedings (Cat. No.01TH8585), Aug 2001, pp. 172-178.

[31] S. Rezaei and R. Sengupta, "Kalman filter-based integration of DGPS and vehicle sensors for localization," IEEE Transactions on Control Systems Technology, vol. 15, no. 6, pp. 1080-1088, Nov. 2007.

[32] K. Liu, H. B. Lim, E. Frazzoli, H. Ji, and V. C. S. Lee, "Improving positioning accuracy using GPS pseudorange measurements for cooperative vehicular localization," IEEE Transactions on Vehicular Technology, vol. 63, no. 6, pp. 2544-2556, Jul. 2014.

[33] X. Zhang, X. Tao, F. Zhu, X. Shi, and F. Wang, "Quality assessment of GNSS observations from an Android N smartphone and positioning performance analysis using time-differenced filtering approach," GPS Solutions, vol. 22, no. 3, p. 70, May 2018. [Online]. Available: https://doi.org/10.1007/s10291-018-0736-8

[34] N. Gogoi, A. Minetto, N. Linty, and F. Dovis, "A controlledenvironment quality assessment of Android GNSS raw measurements," Electronics, vol. 8, no. 1, p. 5, Dec. 2018. [Online]. Available: http://dx.doi.org/10.3390/electronics8010005

[35] U. Robustelli, V. Baiocchi, and G. Pugliano, "Assessment of dual frequency GNSS observations from a Xiaomi Mi 8 android smartphone and positioning performance analysis," Electronics, vol. 8, no. 1, p. 91, Jan. 2019. [Online]. Available: http://dx.doi.org/10.3390/electronics8010091

[36] D. Yoon, C. Kee, J. Seo, and B. Park, "Position accuracy improvement by implementing the DGNSS-CP algorithm in smartphones," Sensors, vol. 16, no. 6, p. 910, Jun. 2016. [Online]. Available: http://dx.doi.org/10.3390/s16060910

[37] P. Dabove and V. D. Pietra, "Towards high accuracy GNSS realtime positioning with smartphones," Advances in Space Research, vol. 63, no. 1, pp. 94 - 102, 2019. [Online]. Available: http://www.sciencedirect.com/science/article/pii/S0273117718306537

[38] L. Hsu, Y. Gu, Y. Huang, and S. Kamijo, "Urban pedestrian navigation using smartphone-based dead reckoning and 3-d map-aided GNSS," IEEE Sensors Journal, vol. 16, no. 5, pp. 1281-1293, Mar. 2016.

[39] Y. Li, Y. Zhuang, H. Lan, P. Zhang, X. Niu, and N. El-Sheimy, "Selfcontained indoor pedestrian navigation using smartphone sensors and magnetic features," IEEE Sensors Journal, vol. 16, no. 19, pp. 7173 7182, Oct. 2016.

[40] A. Štern and A. Kos, "Positioning performance assessment of geodetic, automotive, and smartphone GNSS receivers in standardized road scenarios," IEEE Access, vol. 6, pp. 41 410-41 428, Jul. 2018.

[41] A. Privat, M. Pascaud, and D. Laurichesse, Innovative smartphone applications for Precise Point Positioning. [Online]. Available: https://arc.aiaa.org/doi/abs/10.2514/6.2018-2324

[42] D. Laurichesse, C. Rouch, F. Marmet, and M. Pascaud, "Smartphone applications for precise point positioning," in Proceedings of the ION GNSS, 2017, pp. 171-187.

[43] S. Banville and F. Van Diggelen, "Precise positioning using raw GPS measurements from Android smartphones," GPS World, vol. 27, no. 11, pp. 43-48, 2016.

[44] E. Realini, S. Caldera, L. Pertusini, and D. Sampietro, "Precise GNSS positioning using smart devices," Sensors, vol. 17, no. 10, p. 2434, 2017.

[45] E. D. Kaplan and C. Hegarty, Understanding GPS/GNSS: principles and applications. Artech House, 2017.

[46] R. G. Brown and P. Y. C. Hwang, Introduction to random signals and applied Kalman filtering: with MATLAB exercises. John Wiley \& Sons, Inc., 2012.

[47] R. Zekavat and R. M. Buehrer, Handbook of position location: Theory, practice and advances. John Wiley \& Sons, 2011, vol. 27.

[48] A. Minetto and F. Dovis, "On the information carried by correlated collaborative ranging measurements for hybrid positioning," IEEE Transactions on Vehicular Technology, pp. 1-1, Dec. 2019.
[49] Y. Shen, H. Wymeersch, and M. Z. Win, "Fundamental limits of wideband localization part II: Cooperative networks," IEEE Transactions on Information Theory, vol. 56, no. 10, pp. 4981-5000, Oct. 2010.

[50] E. Steinmetz, R. Emardson, F. Brännström, and H. Wymeersch, "Theoretical limits on cooperative positioning in mixed traffic," IEEE Access, vol. 7, pp. 49712-49 725, Apr. 2019.

[51] F. de Ponte Müller, A. Steingass, and T. Strang, "Zero-baseline measurements for relative positioning in vehicular environments," in 6th European Workshop on GNSS Signals and Signal Processing, 2013.

[52] M. S. Sheijani, A. Gholami, N. Davari, and M. Emami, "Implementation and performance comparison of indirect Kalman filtering approaches for AUV integrated navigation system using low cost IMU," in $201321 s t$ Iranian Conference on Electrical Engineering (ICEE). IEEE, 2013, pp. $1-6$.

[53] MongoDB. (2019) MS Windows NT kernel description. [Online]. Available: https://www.mongodb.com/

[54] F. Van Diggelen, "Innovation: GPS accuracy-lies, damn lies, and statistics," GPS WORLD, vol. 9, pp. 41-45, 1998.

[55] P. Misra and P. Enge, Global Positioning System: Signals, Measurements and Performance Second Edition. Lincoln, MA: Ganga-Jamuna Press, 2006.

[56] N. Gogoi, A. Minetto, and F. Dovis, "On the cooperative ranging between Android smartphones sharing raw GNSS measurements," in Proceedings of VTC2019-Fall Honolulu Intelligent Connection and Transportation), September 2019.

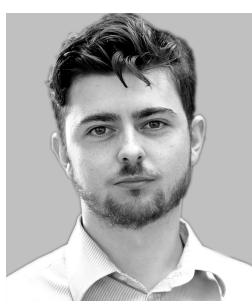

Alex Minetto (GS'17-M'20) was born in Pinerolo, Italy, in 1990. He received the B.Sc. and M.sc. degrees in Telecommunications Engineering from Politecnico di Torino, Turin, Italy and his Ph.D. degree in Electrical, Electronics and Communications Engineering, in 2020. He joined the Department of Electronics and Telecommunications of Politecnico di Torino in 2019 as research and teaching assistent. His current research interests cover signal processing and advanced Bayesian estimation applied to Global Navigation Satellite System (GNSS) cooperative re-

ceivers.

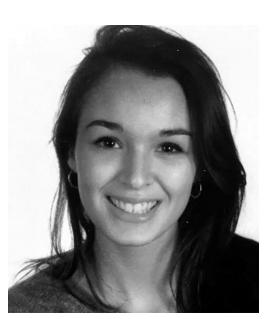

Maria Chiara Bello was born in Turin, Italy, in 1995. She received her M.Sc. degree in Information and Communication Technologies for Smart Societies from Politecnico di Torino, in 2019. She was with the Department of Electronics and Telecommunications as research assistant at for the NavSAS research group at LINKS foundation, Turin, Italy. Her research interests cover GNSS navigation and positioning algorithms, and Bayesian estimation.

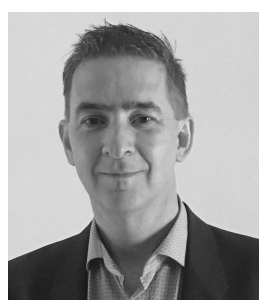

Fabio Dovis (GS'98-M'01) was born in Bruino, Italy,in 1970. He received his M.Sc. degree in 1996 and his Ph.D. degree in 2000, both from Politecnico di Torino, Turin, Italy. He joined the Department of Electronics and Telecommunications of Politecnico di Torino as an assistant professor in 2004 and since 2014 he is associate professor in the same department where he coordinates the Navigation Signal Analysis and Simulation (NavSAS) research group. $\mathrm{He}$ serves as a member of the IEEE Aerospace and Electronics Systems Society Navigation Systems Panel. His research interests cover the design of GPS and Galileo receivers and advanced signal processing for interference and multipath detection and mitigation, as well as ionospheric monitoring. 